\title{
Role of Nr13 in regulation of programmed cell death in the bursa of Fabricius
}

\author{
Ruey-min Lee, ${ }^{1,4}$ Germain Gillet, ${ }^{2}$ Joan Burnside, ${ }^{3}$ Sandra Jo Thomas, ${ }^{1}$ and Paul Neiman ${ }^{4,5}$ \\ ${ }^{1}$ Fred-Hutchinson Cancer Research Center, Seattle, Washington 98109 USA; ${ }^{2}$ Institut de Biologie et de Chimie \\ der Proteines, 69376 Lyon Cedex 07, France; ${ }^{3}$ Department of Animal and Food Sciences, University of Delaware, Newark, \\ Delaware 19717 USA; ${ }^{4}$ Departments of Medicine and Pathology, University of Washington, Seattle, Washington 98109 USA
}

Apoptotic cell death is developmentally regulated in the chicken bursa of Fabricius. Although apoptosis is low in the embryonic bursa, cell death increases markedly after hatching. The expression of Bcl2 family cell death antagonists was examined to identify the genes that regulate bursal cell apoptosis. The expression of $\mathrm{Bcl}-\mathrm{xL}$, A1, and Mcl1 was detected in both embryos and hatched birds, whereas Nr13 was expressed at high levels in embryonic bursa, and decreased significantly after hatching, correlating inversely with apoptosis. The oncogene v-reland phorbol myristate acetate, two known inhibitors of bursal cell apoptosis, induced Nr13 expression. Overexpression of Nr13 in DT40 bursal lymphoma cells protected them from low serum-induced apoptosis. The mechanism of inhibition of apoptosis by Nr13 is likely to involve a critical BH4 domain and interaction with death agonist Bax. Deletion of the BH4 domain converted Nr13 into a death agonist. Bax coimmunoprecipitated with Nr13 and Bax was induced, whereas Nr13 levels diminished when bursal lymphoblasts were induced to apoptosis by dispersion. Bursal transplantation studies demonstrated that Nr13 could prevent the in vivo programmed elimination of bursal stem cells after hatching, suggesting that Nr13 plays a role in maintaining bursal stem cells.

[Key Words: Nr13; apoptosis; bursa; transplant; stem cells]

Received November 13, 1998; revised version accepted January 19, 1999.

Apoptosis (programmed cell death) is a critical mechanism for maintaining biological homeostasis. Morphologically, it is characterized by nuclear fragmentation, chromatin condensation, cytoplasmic membrane blebbing, and mitochondrial disintegration (Wyllie et al. 1980). Using this mechanism, organisms are able to eradicate unnecessary cells, both during development and throughout the subsequent life span. Abnormal regulation of this mechanism leads to the development of neoplasms, and autoimmune and degenerative diseases (for review see Rudin and Thompson 1997). During the past several years, significant progress has been made in identifying the molecular determinants of apoptosis (for review see Yang and Korsmeyer 1996; Reed 1997; Cryns and Yuan 1998). Specifically, a number of cell death agonists and antagonists have been identified. Bcl2, Bcl-xL, A1, Mcl1, Nr13, and CED9 are the death antagonists of the Bcl2 family. Bax, Bcl-xS, Bad, Bak, and Bik are the death agonists sharing homology with other members of the Bcl2 family. Genetic and biochemical studies of three proteins of the central pathway in Caenorhabditis elegans, CED3, CED4, and CED9 (Horvitz et

${ }^{5}$ Corresponding author.

E-MAIL pneiman@fhcrc.org; FAX (206) 667-6523. al. 1994; Chinnaiyan et al. 1997), have provided seminal insights into underlying molecular mechanisms. However, exactly how Bcl2 family members protect or promote cell death remains to be defined (Reed 1997). They appear to mediate their functions through homodimer or heterodimer formation, and they interact with other proteins in critical signal transduction pathways. Members of the Bcl2 family interact and prevent the activation of downstream caspases, a group of proteases that cleave at aspartic acid residues and promote cell death (Cryns et al. 1997; Nicholson and Thornberry 1997). Other reported Bcl2 functions include alteration of redox potential, calcium permeability, and protein transport (Kroemer 1997). The solution of the structure of Bcl-xL suggests that members of the Bcl2 family may function through membrane channel formation (Muchmore et al. 1996). The release of cytochrome $c$ from mitochondria into the cytosol during apoptosis is taken as evidence in support of this theory (Jurgensmeier et al. 1998; Rosse et al. 1998).

The chicken bursa of Fabricius is the lymphoid organ responsible for avian B-cell development (for review see Weill and Reynaud 1987). Apoptosis appears to play a major role in the developmental process of bursa (Neiman et al. 1991). The chicken bursa is composed of 
$\sim 10^{4}$ follicles, which contain surface IgM-positive lymphocytes distributed within the cortex and medulla. Bursal development begins at day 10 of embryogenesis with migration of hematopoietic mesenchymal cells to the bursal anlage. At about day 15, stem cells enter the developing follicles and give rise to the IgM-positive B-cell population. A gene conversion mechanism is used for preimmune immunoglobulin gene diversification within the embryonic follicular population (Reynaud et al. 1987; Thompson and Neiman 1987). However, only a small proportion of the bursal B cells complete maturation and leave the bursa. The majority of the cells undergo programmed cell death in the bursal follicles (Paramithiotis and Ratcliffe 1993; Jacobsen et al. 1996). Apoptosis is particularly prominent in bursal follicles after hatching (Neiman et al. 1994b), and embryonic and posthatching bursal lymphocytes can be induced to undergo apoptosis by loss of cell contact (Neiman et al. 1991). Embryonic bursal follicles contain a transplantable stem cell compartment, comprised of $1 \%$ or less of the bursal population (Toivanen and Toivanen 1973; Pink et al. 1985), which is also subject to programmed elimination after hatching. It has been demonstrated that there are no transplantable bursal stem cells left in the bursa by 4 weeks after hatching (Thompson and Neiman 1987).

We investigated the expression of bcl2 family genes during B-cell development in the bursa to identify regulators of this extensive cell death activity in the bursa. Previous studies (Eguchi et al. 1992) and our own unpublished results indicated that $b c l 2$ itself was expressed at a very low level, if at all, in the bursa. Therefore, we examined the expression of other family members. Our results suggest that $\mathrm{Nr} 13$ is an important protein for bursal B-cell viability and programmed survival of bursal stem cells.

\section{Results}

Expression of antagonists of apoptosis in bursal development

The expression of Bcl2 members of death antagonists was first investigated to determine which member of the family correlates with bursal cell survival. We analyzed expression of other death antagonists in the Bcl2 family, including Bcl-xL, Nr13, A1, and Mcl1. Both A1 and mcl1 are hematopoiesis-related genes able to protect hematopoietic cells from interleukin-3 withdrawal-induced apoptosis (Lin et al. 1996; Zhou et al. 1997). Nr13 is a relatively newly identified member of the Bcl2 family, initially identified as a v-src-activated gene in developing neural tissues, with a pattern of membrane localization similar to that of Bcl2 (Gillet et al. 1995). Ectopic overexpression of quail Nr13 protects cultured mammalian hematopoietic cells against factor withdrawal-induced apoptosis (Mangeney et al. 1996).

The expression of these four genes was analyzed by Northern analysis. Nr13 RNA was present in bursal follicles at days 15,17 , and 21 of embryogenesis, but was no longer present 28 days after hatching (Fig. 1). Mcl1 RNA

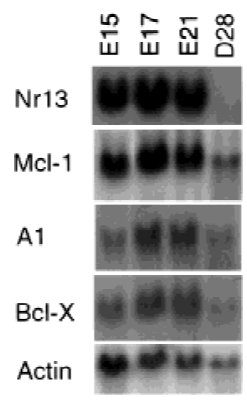

Figure 1. Northern analysis of Bcl2 family in the bursa. Total cellular RNAs (15 $\mu \mathrm{g})$ from embryonic days 15, 17, 21, and day 28 after hatching bursa were analyzed with probes of Nr13, $\mathrm{Mcll}, \mathrm{A} 1, \mathrm{Bcl}-\mathrm{X}$, and $\beta$-actin at specific activity of $2 \times 10^{6} \mathrm{cpm} /$ $\mathrm{ml}$. The exposure time was $16 \mathrm{hr}$ for Nr13 and Mcl1, $72 \mathrm{hr}$ for $\mathrm{Al}$ and Bcl-X, and $8 \mathrm{hr}$ for actin.

was present in embryonic bursa in amounts comparable to Nr13. Mcl1 RNA decreased somewhat after hatching (when compared with the actin-loading control), but did not disappear at day 28 after hatching like Nr13. Finally, A1 and Bcl-x are both present at much lower levels than Mcl1 and Nr13, requiring three times longer exposure of the Northern blot than did Nr13 and Mcl1 Northern blots, and showing minimal change after hatching compared with the loading control (Fig. 1). We found the pattern of Nr13 expression particularly interesting, as Nr13 RNA was detected in embryonic bursa but not in normal bursa after hatching. We also failed to detect by immunoblotting Nr13 in bursa after hatching (Fig.7a, below). This pattern correlated inversely with the intensity of bursal apoptosis and the programmed elimination of stem cells. For these reasons, we chose Nr13 for further study.

Induction of apoptosis in bursal cells by dispersion causes an increase of Bax and a decrease of Nr13

Bursal cells require cell-cell interaction to maintain their viability. When embryonic bursal cells are dispersed, they rapidly undergo apoptosis, and can become terminal deoxynucleotide end-labeling (TUNEL) positive within an hour (Neiman et al. 1991). We dispersed day 21 embryonic bursal cells by passing through nylon mesh as described (Neiman et al. 1991) to trigger apoptosis. Proteins were extracted at different time points after dispersion for Western blot analysis. The levels of Nr13 decreased by $2 \mathrm{hr}$ after dispersion (Fig. 2a). The levels of Bax, a death agonist, were analyzed by Western blot, and the Bax levels increased after $1 \mathrm{hr}$ of dispersion (not shown). As shown in Figure 2b, we repeated this analysis to study the kinetics of Bax increase. Bax was not detectable in undispersed bursa, but appeared by 30 min after bursal cell dispersion and continued to increase for at least $2 \mathrm{hr}$. Although Bax was reported to be a transcriptional activation target of p53 (Miyashita and Reed 1995), this induction of Bax with dispersion of bursal cells is probably independent of p53, because p53 pro- 
Lee et al.

a

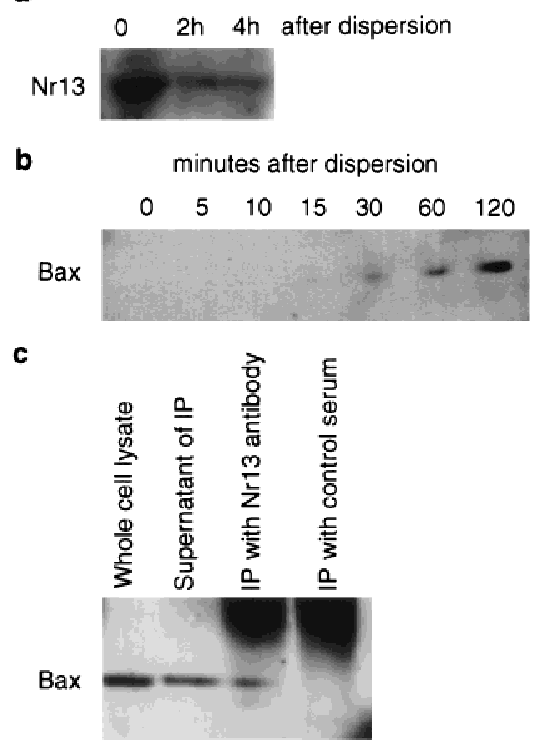

Figure 2. (a) Western analysis of Nr13 in bursa after dispersion. Bursas from newly hatched chickens were dissected and dispersed. Cells were harvested 2 and $4 \mathrm{hr}$ after dispersion for Western blot analysis. A total of $80 \mu \mathrm{g}$ of protein (by Bio-Rad protein assay) was loaded into each lane. (b) Kinetic study of Bax increase during bursal cell dispersion. Samples were taken at different times after bursal dispersion and $80 \mu \mathrm{g}$ of protein was analyzed by Western blot analysis with Bax antiserum. (c) Western blot analysis of Nr13 immune complex with Bax antiserum. Immunoprecipitation was performed with anti-Nr13 antibody as described in Materials and Methods. Immunoprecipitates (IP) were separated by SDS-PAGE and the blot was probed with anti-Bax antibody.

tein and mRNA levels are not altered by the dispersion process (Neiman et al. 1994a). We conclude that the ratio of Nr13 to Bax decreases dramatically after dispersion of bursal lymphocytes.

Induction of Nr13 by v-rel and phorbol ester, two known inhibitors of bursal apoptosis

Phorbol myristate acetate (PMA) is known transiently to inhibit dispersion-induced bursal apoptosis (Asakawa et al. 1993; Compton and Waldrip 1998). We tested a chicken bursal lymphoma-derived cell line, DT40 (Baba et al. 1985), to determine whether PMA could induce Nr13 expression. Nr13 RNA increased within $1 \mathrm{hr}$ of PMA treatment and continued to increase at least until $6 \mathrm{hr}$ (Fig. 3a). PMA induction of Nr13 was also observed in dispersed embryonic bursal cells (Fig. 3b). These results suggest that Nr13 could be an intermediate in PMA-induced protection from cell death.

Another known inhibitor of bursal cell apoptosis is proto-oncogene v-rel, a member of the NF- $\mathrm{KB}$ transcription activators. v-rel can transform bursal cells in vivo and block apoptosis induced by bursal dispersion (Neiman et al. 1991; White et al. 1995). We constructed retroviral vectors by cloning v-rel (White and Gilmore

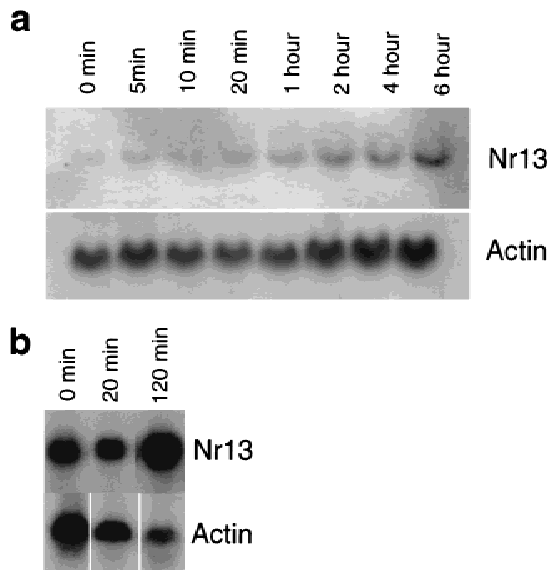

Figure 3. Activation of Nr13 by PMA in bursa-derived DT40 cells and dispersed bursa. (a) DT40 cells were incubated with $100 \mathrm{ng} / \mathrm{ml}$ PMA and were harvested at different time points for RNA analysis. Northern blot was probed with $\mathrm{Nr} 13$ and $\beta$-actin cDNA probes. (b) Dispersed embryonic bursal cells were incubated with $100 \mathrm{ng} / \mathrm{ml}$ PMA and were harvested 20 and $120 \mathrm{~min}$ after dispersion for Northern blot analysis. Northern blot was probed with $\mathrm{Nr} 13$ and $\beta$-actin cDNA probe.

1993) into the LXSN vector (Fig. 4a) and infected DT40 cells with v-rel vectors to test whether v-rel would affect Nr13 expression. After the cells were selected with G418, the control DT40 cells and the DT40 cells infected with v-rel were incubated at various temperatures to examine their growth and effects on Nr13 expression. The growth rate of DT40 cells was not affected by v-rel at $37^{\circ} \mathrm{C}, 40^{\circ} \mathrm{C}$, and $42^{\circ} \mathrm{C}$ (not shown). Northern blot analysis demonstrated that Nr13 mRNA increased

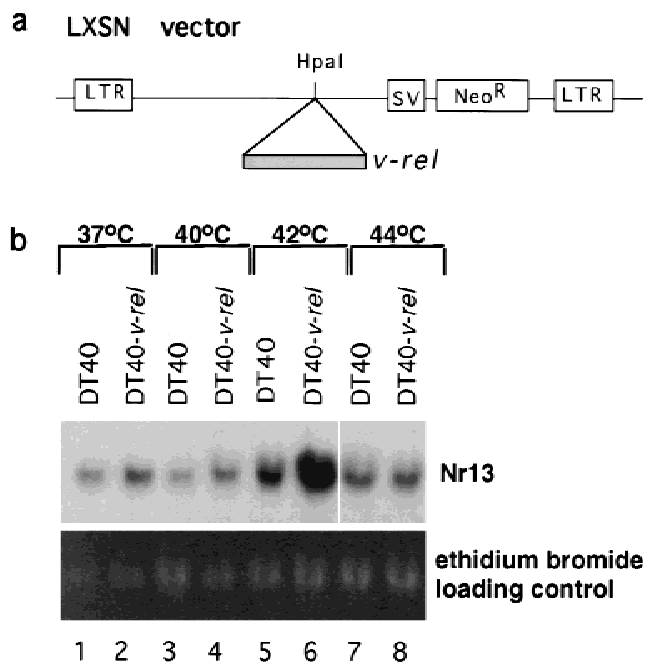

Figure 4. Induction of $\mathrm{Nr} 13$ by v-rel. (a) Retroviral construct of the v-rel-expressing vector. (b) Northern blot analysis of Nr13 expression in DT40 cells at different temperatures. DT40 cells, DT40 cells expressing v-rel were incubated at $37^{\circ} \mathrm{C}, 40^{\circ} \mathrm{C}, 42^{\circ} \mathrm{C}$, and $44^{\circ} \mathrm{C}$. (Top) RNAs were isolated and probed with labeled Nr13 probe. (Bottom) The ethidium bromide staining was used as the loading control. 
threefold in DT40 cells when the temperature was shifted from $37^{\circ} \mathrm{C}$ to $42^{\circ} \mathrm{C}$ (near the physiological body temperature of chicken) (Fig. 4b, lanes 1, 5). Nr13 RNA was only slightly increased at $37^{\circ} \mathrm{C}$ by v-rel (Fig. $4 \mathrm{~b}$, lanes 1,2 ) but was significantly enhanced by v-rel at $42^{\circ} \mathrm{C}$ (Fig. $4 \mathrm{~b}$, lanes 5,6$)$. At $44^{\circ} \mathrm{C}$ the growth rate of the DT40 cells was impaired (not shown) and no effect of v-rel on $\mathrm{Nr} 13$ RNA was observed. These results suggest that rel-related Nr13 activation may contribute to v-rel-induced inhibition of apoptosis.

Overexpression of Nr13 in DT40 cells protects DT40 cells from serum withdrawal-induced apoptosis

We then tested the DT40 cell line to determine whether increased levels of Nr13 could inhibit apoptosis in these cells. DT40 cells express constitutively high levels of c-myc as a result of retroviral promoter insertion (Hayward et al. 1981). Like previously reported myc-overexpressing Rat1 cells (Evan et al. 1992), serum deprivation of DT40 cells suppresses net cell growth in the culture as a result of increased apoptosis. We studied whether Nr13 could protect DT40 cells from serum withdrawal-induced apoptosis. As shown in Figure 5a, Nr13 retroviral vectors LNRSN and LNRCG were constructed in pLXSN (neomycin resistance expressing) and pLXCG [green fluorescence protein (GFP) expressing] plasmids, and PG13 packaging lines expressing these viruses were prepared (Miller and Rosman 1989; Miller et al. 1991). The DT40 cells have a low but detectable level of Nr13 expression. After infection with the LNRSN virus and selection with G418, DT40 cells expressed at least a 5- to 10-fold increase of Nr13 protein (Fig. 5b). A similar increase of Nr13 was observed in DT40 cells infected with LNRCG virus and sorted for GFP-positive cells by flow cytometry (not shown). In standard media [10\% bovine calf serum (BCS) and $2 \%$ chicken serum (CS)] parental wild-type DT40 cells grew for 3 days and leveled off. Under reduced serum condition (1\% CS and no BCS), both wild-type DT40 cells and DT40-overexpressing Nr13 cells were able to grow at similar rate for 2 days. Net growth of wild-type DT40 cells ceased after 2 days, and there was net cell death attributable to apoptosis, whereas the growth of Nr13-overexpressing cells was closer to that of DT40 in normal serum (Fig. 5c). This experiment indicated that Nr13 can protect bursa-derived cells against serum withdrawal-enhanced apoptosis in the presence of elevated Myc.

\section{Potential mechanisms of Nr13 function}

Nr13 interacts with Bax Bax is a death-promoting agonist that interacts physically with $\mathrm{Bcl} 2$ and $\mathrm{Bcl}-\mathrm{xL}$ (Oltvai et al. 1993). Bax-deficient mice have the phenotype of lymphoid hyperplasia in both $\mathrm{T}$ and $\mathrm{B}$ lineages (Knudson et al. 1995). Quantitative analysis in IL-3-dependent FL5.12 cells showed that, when half or more of endogenous Bax was heterodimerized with $\mathrm{Bcl} 2$ or Bcl$\mathrm{xL}$, apoptosis induced by IL-3 withdrawal was repressed

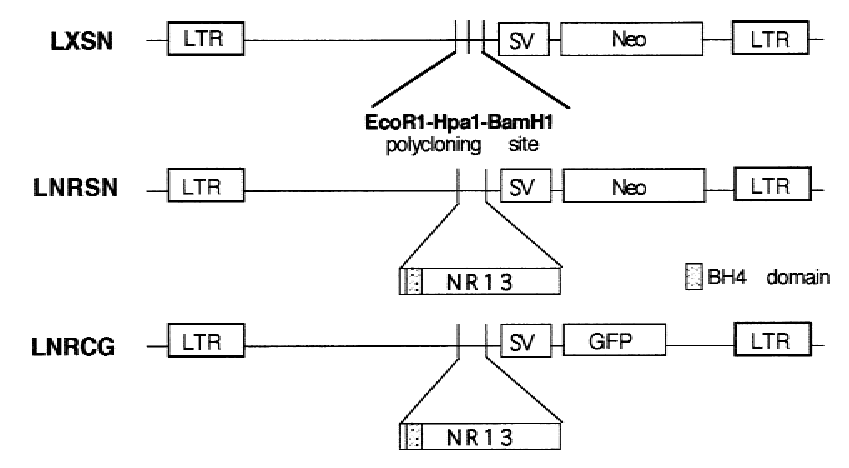

b

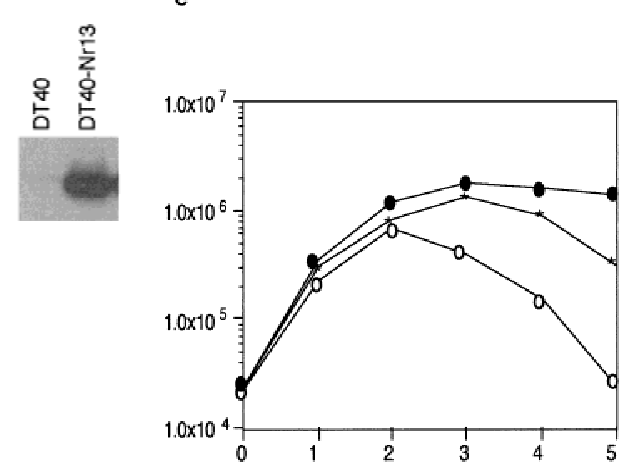

Figure 5. Protection of apoptosis by overexpression of Nr13. (a) Retroviral constructs for Nr13 overexpression. (b) Western blot analysis of DT40 cells and DT40 cells infected with Nr13 retroviral vectors. $\mathrm{Nr} 13$ retroviral infection results in 5- tol0-fold increase of Nr13. (c) Growth curve of DT40 cells in standard 2\% CS, $10 \%$ BCS condition $(\bullet)$, and $1 \%$ CS condition $(O)$, and DT40 cells overexpressing Nr13 (DT40/Nr13) in 1\% CS condition (*). Cell count was performed with trypan blue exclusion.

(Yang et al. 1995). On the other hand, when Bax was displaced from Bcl2 or Bcl-xL as a result of binding of Bcl2 and Bcl-xL by Bad, another Bcl2 family death agonist, cell death was promoted (Yang et al. 1995). Therefore, cell survival or death is thought to be dependent on the interaction between Bcl2/Bcl-xL and Bax. Both Bax and Nr13 can be detected in DT40 cells, thus, we investigated whether Bax interacts with Nr13 by coimmunoprecipitation. Cell lysates from DT40 were immunoprecipitated with Nr13 antisera and the immunoprecipitates were analyzed by Western immunoblotting using Bax antisera. Bax was detected in the whole cell lysate, the supernatant of the immunoprecipitate, and the Nr13 immunoprecipitate, but not in control immunoprecipitate with normal rabbit serum (see Fig. 2c). This experiment suggests that a portion of Bax is complexed with Nr13 in DT40 cells, and that Nr13 may control apoptosis in bursal cells by interaction with Bax. When the level of Bax increases relative to the level of Nr13, for example 1 hr after bursal dispersion, bursal cells undergo apoptosis.

BH4 domain of Nr13 is required for protection of cell death and deletion of BH4 domain converts Nr13 into a death agonist Four conserved homology domains of 
Lee et al.

the Bcl2 family have been defined: $\mathrm{BH} 1, \mathrm{BH} 2, \mathrm{BH} 3$ and BH4 (Hanada et al. 1995; Reed 1997). The BH1 and BH2 domains are known to be essential for homodimer or heterodimer formation. BH3 plays the role of 'ligand' during heterozygous dimerization of $\mathrm{Bcl} 2$ family members. The $\mathrm{BH} 4$ domain is an amino-terminal $\alpha$-helix that appears to play a regulatory role by docking of Raf1 (Wang et al. 1996a), Bag1 (Wang et al. 1996b), calcineurin (Shibasaki et al. 1997), or Ced4/Apaf1 (Chinnaiyan et al. 1997). It was recently reported that the $\mathrm{BH} 4$ domain of $\mathrm{Bcl} 2$ has a caspase cleavage site at its carboxy-terminal end that can be cleaved by a caspase, converting Bcl2 into a Bax-like death effector (Cheng et al. 1997). We analyzed the predicted secondary structure of $\mathrm{Nr} 13$ and compared it with Bcl2 by NNPREDICT, a protein secondary structure prediction program (Kneller et al. 1990).
We found that Nr13 also has seven predicted $\alpha$-helices similar to Bcl2 (Fig. 6a). However, unlike Bcl2 or Bcl-xL, $\mathrm{Nr} 13$ has a relatively short loop between the amino-terminal helices. Moreover, no clear sequence homology to the amino-terminal $\mathrm{BH} 4$ domain of $\mathrm{Bcl} 2$ could be identified in $\mathrm{Nr} 13$, and a caspase cleavage site (aspartic acid) was not found. There is, however, an apparent $\alpha$-helix at the Nr13 amino-terminus that could be the potential counterpart of the $\mathrm{BH} 4$ domain. This portion of $\mathrm{Nr} 13$ sequence was deleted and tested to determine the function of this BH4-like helix. The Nr13 deletion mutant was cloned into pLXSN to generate a retroviral vector, and DT40 cells were infected and selected for G418 resistance as was previously done with full-length Nr13. Because available antibodies to full-length Nr13 did not react with the deletion mutant protein, the expression of

a

Chicken Bcl-2

MAHPGRRGYDNREIVL.KYTHYKLSQRGYDWAAGEDRPPVPPAPAPAAAPAAVAAAGASSHHRPEPPGSAAAS

EVPPAEGLRPAPPGVHLALRQAGDEFSRRYQRDFAOMSGQLHLTPFTAHGRFVAVVEELFRDGVNWGRTVAF

FEFGGVMCVESVNREMSPLVDNIATWMTEYLNRHLHNWIQDNGGWDAFVELYGNSMRPLFDF SWISLRTIISS

LVLVGACITLGAYLGEK

SECONDARY STRUCTURE PREDICTION(H $=$ helix, E = strand, - = no prediction)

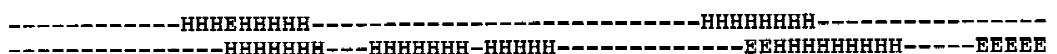

-

HEE--EEERHHHH----

Chicken Nr13

MPGSLREETALLLEDYFOHRAGGAALPPSATAAELRRAAAELERRERPFFRSCAPLARAEPREAAALILRK BH4

VAAQLETDGGLNWGRLIALVVFAGTLAAALAESACEEGP SRTAAALTAYLAEEQGEWMEEHGGWDGFCRF

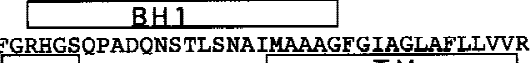

FGRHGSOPADQNS TLSNA IMAAAGFGIAGLAFLLVVR

SECONDARY STRUTURE PREDICTION(B = helix, E = strand, - = no prediction)

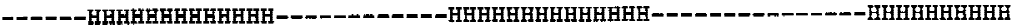

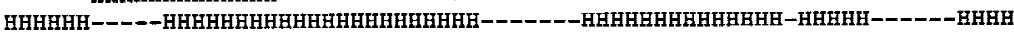

E-C-1--

b

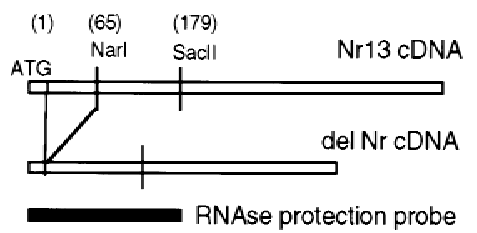

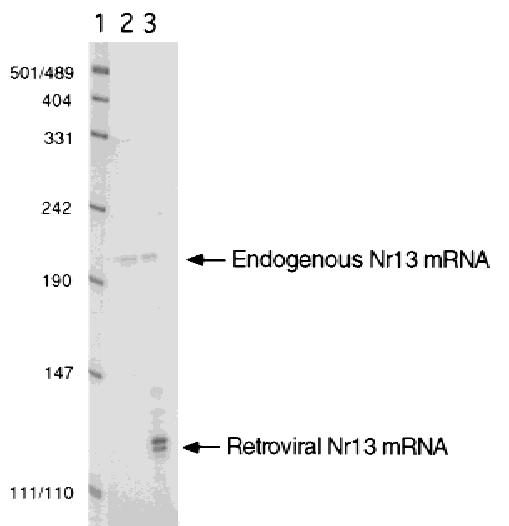

C

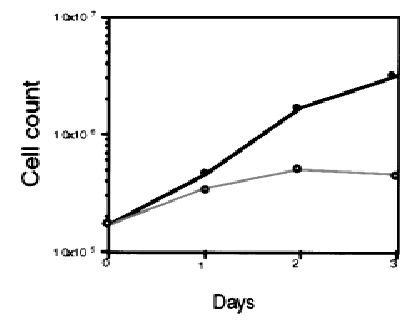

Figure 6. BH4 domain of Nr13. (a) Predicted secondary structures of chicken Bc12 and Nr13. The primary sequences and the domain structures are shown at top. The predicted secondary structures are shown on the bottom. $(\mathrm{H})$ helix; $(-)$ no prediction; $(\mathrm{E}) \beta$ strand. $(b)$ Confirmation of expression of the truncated mutant by RNase protection assay. Deletion between ATG initiation codon and NarI site was generated as described in Materials and Methods. The probe for RNase protection assay is shown at left. The first lane of the gel is the marker and the size of each marker is indicated. The second and third lanes are DT40 cells and DT40 cells infected with truncated mutant vectors. (c) Growth curves of DT40 cells and DT40 expressing amino-terminal truncated mutant in $1 \%$ CS media.

(-) DT40 1\% CS; (O) DT40-del-Nr 1\% CS. Cell count was performed with trypan blue exclusion. 
truncated Nr13 was confirmed by a RNase protection assay (Fig. 6b). In this assay, endogenous Nr13 mRNA produced a 190-base RNA fragment, and the retroviral mRNA resulted in a 120-base RNA fragment. The signal from retroviral mRNA was also at least three fold more intense than that from the endogenous mRNA, indicating overexpression from the retroviral promoter. Phenotypically, the DT40 cells expressing the deletion Nr13 mutant have a normal growth curve under standard growth conditions in $10 \%$ BCS and $2 \%$ CS. However, when treated with $1 \%$ CS media, the amino-terminal deletion mutant-expressing cells were more susceptible than wild-type DT40 cells to serum withdrawal-induced cell death (Fig. 6c). Thus, the BH4 domain of $\mathrm{Nr} 13$ is essential for the inhibition of apoptosis under these conditions, and loss of the $\mathrm{BH} 4$ domain appeared to convert Nr13 into a death agonist.

\section{Nr13 protects bursal stem cells from programmed elimination}

The in vivo function of Nr13 was investigated using a bursal transplant model established in this laboratory. Donor bursal cells from unhatched embryonic bursa can reconstitute recipient birds treated with cyclophosphamide, which ablates the lymphoid population of bursal follicles. Nr13 was introduced into donor cells by ex vivo cocultivation with LNRSN vectors for expression in reconstituted bursal follicles and their progeny. Bursal transplantation was performed as previously described (Neiman et al. 1985; Thompson et al. 1987) except that day 15 syngenic embryonic bursal cells were used as donors and cyclophosphamide-treated day 18 embryos were used as recipients. The transplanted birds were sacrificed at 4 weeks after hatching. Reconstituted bursal follicles appeared normal on routine histological examination. Western blot analysis detected Nr13 expression in reconstituted follicles compared with normal 4-week bursa, which lacks detectable Nr13 (Fig. 7a). The primary transplanted bursal cells 4 weeks after hatching were used as donors for a secondary transplantation experiment into cyclophosphamide-ablated recipient birds. Histological examination of the secondary transplant bursas revealed that $10-55 \%$ of the follicles were reconstituted and appeared normal (Fig. 7b, middle, and c). Bursas from either cyclophosphamide-ablated controls or five control transplants using donor cells from 4-week normal bursa resulted in empty follicles (Fig.7b, left, and c), consistent with previous reports demonstrating absence of stem cells in bursa after hatching (Toivanen and Toivanen 1973; Pink et al. 1985). Western blot analysis confirmed that Nr13 was detected in secondary reconstituted follicles transduced with LNRSN (Fig. 7a). The reconstitution of follicles and detection of Nr13 in secondary transplant assays suggested that Nr13 induced persistence of stem cells. We concluded that Nr13 overexpression in bursal stem cells maintained the viability of the transplantable stem cells after hatching. To confirm this observation we repeated this transplantation experiment using the LNRCG vector (see Fig. 5a) to in- fect 15-day-old embryonic donor stem cells, which were then transplanted into cyclophosphamide-ablated primary recipients. Four weeks after hatching we again used the reconstituted bursal follicle population to transplant secondary recipients. The secondary reconstituted follicles demonstrated cells positive for green fluorescence protein (Fig.7b, right), confirming the ability of Nr13 to preserve the viability of bursal stem cells.

The transplant experiment with the GFP marker also allowed us to examine the effects of Nr13 on the differentiated progeny of transplanted bursal stem cells. The reconstituted follicles were normal in size and dispersion of the secondary follicles still induced a similar degree of apoptosis as in normal bursal lymphocytes 4 weeks after hatching (data not shown). This result suggested that constitutive expression of Nr13 from our viral vectors had a selective effect on the small transplantable stem cell compartment, which was protected from programmed elimination, but did not markedly influence cell death in the progeny of these cells. We then analyzed the differentiation and migration of Nr13- expressing bursal cells. Peripheral blood buffy coats were isolated from secondary transplant birds, and B -cells were identified by monoclonal antibody against chicken immunoglobulin light chain by flow cytometry. Three LNRCG secondary birds showed $10 \%, 14 \%$ and $63 \%$ of Ig $\lambda$-positive B cells that were also GFP positive. Essentially all GFP positive cells in peripheral blood were Ig $\lambda$ positive in these three birds (data not shown). These results indicated that Nr13-expressing bursal cells can differentiate to mature B cells, which can migrate from the bursa.

\section{Discussion}

\section{Role of Nr13 in bursal cell survival}

Our studies indicate that several members of the Bcl2 family were expressed in the bursa. Nr13 was emphasized in this study based on the fact that, among the several expressed antagonists of apoptosis, Nr13 showed the largest decrease in expression after hatching. This pattern of expression correlates both with the intensity of apoptosis in the bursal population and the developmental regulation of bursal stem cells, which are eliminated after hatching. Constitutive Nr13 expression from retroviral vectors in bursal stem cells did, in fact, protect them from programmed elimination and did not interfere with their migration and maturation into peripheral B cells. This result implies that down-regulation of Nr13 is involved in the programmed elimination of bursal stem cells. In contrast, spontaneous and dispersion-induced apoptotic cell death in the progeny of these stem cells was not markedly influenced by persistent expression of Nr13. This result is, perhaps, not surprising given the multiplicity of apoptotic regulators we and others (Jacobsen et al. 1996) have detected in the bursa. Hence, there may be compensatory mechanisms to maintain cell death homeostasis in the progeny of bursal stem cells despite abnormal expression of a single component 
Lee et al.

a
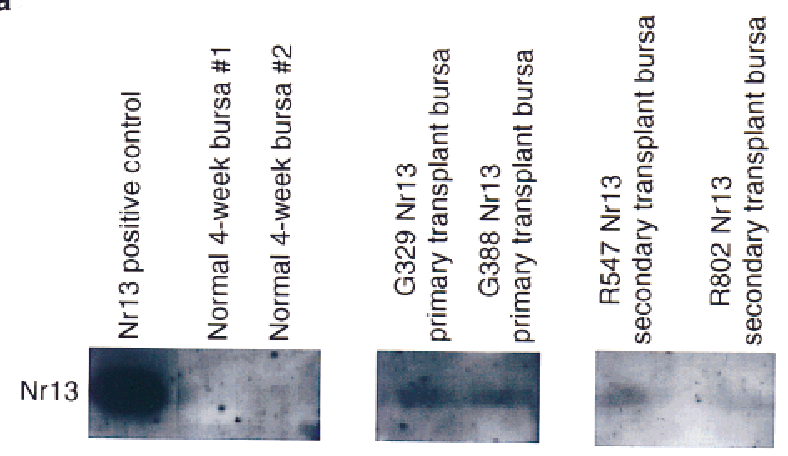

b.
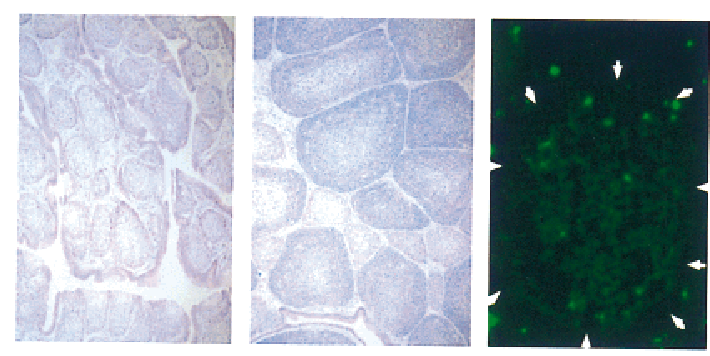

C.

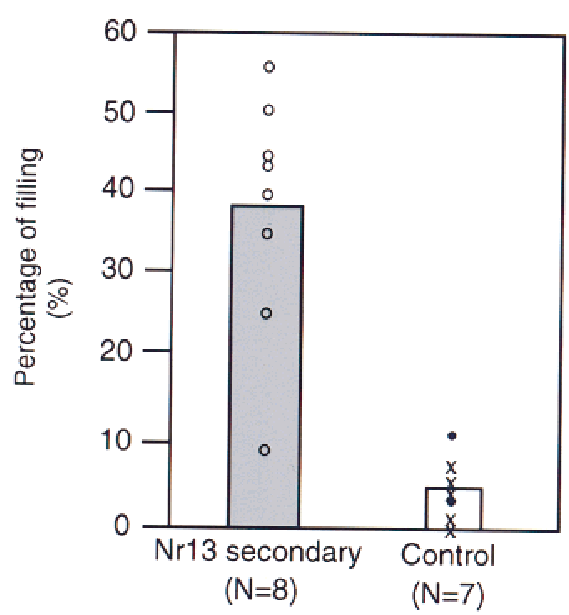

Figure 7. Expression of $\mathrm{Nr} 13$ in bursal transplantation. (a) Western blot analysis of 4-week old normal bursa, two primary and two secondary transplanted bursas with donor bursal cells infected with LNRSN vector. The Nr13-positive control is from DT40 cells infected with LNRSN vector. (b) Histological examination of bursa from a control transplantation with normal 4-week-old donor shows only empty follicles (left), whereas a Nr13-expressing secondary reconstituted bursa shows both empty and reconstituted follicles (middle) (both in original magnification 100×). (Right) Expression of GFP in a reconstituted follicle of LNRCG secondary transplantation (original magnification 250x). The boundary of a reconstituted follicle is indicated by arrowheads. (c) Percentage of filling in $\mathrm{Nr} 13$ expression secondary transplant bursa (five LNRCG secondary transplant bursas and three LNRSN transplant bursas) (O) and control, which includes two birds with cyclophosphamide-only control (O) and five birds transplanted with donor cells from normal 4-week-old bursa (cross). The percentage of filling was calculated by numbers of filled follicles divided by numbers of total follicles. of the cell death pathway. Nevertheless, as suggested by responses to phorbol ester and v-rel, Nr13 may play a role in the essential survival machinery in many types of bursal cells. Moreover, Nr13 may be central and essential to survival in the stem cell compartment, and perhaps in tumor cells derived from bursal stem cells. DT40 is derived from a bursal lymphoma induced by myc oncogene overexpression in bursal stem cells (Baba et al. 1985). We did obtain evidence that survival of these cells, at least in culture, was markedly influenced by $\mathrm{Nr} 13$, being enhanced by overexpression and diminished by a BH4 deletion mutation of Nr13.

\section{Nr13 and Bax}

Bax is a death agonist thought to function in part by interacting with and preventing $\mathrm{Bcl} 2$ or its homologs from binding with the CED4 homolog, Apaf1 (Oltvai et al. 1993; Sedlak et al. 1995). This interaction allows Apaf1 to activate a caspase cascade and induce cell death. Bax is also thought to trigger apoptosis by its pore forming activity (Schlesinger et al. 1997), which is also blocked by Bcl2. We used dispersion as a model to induce bursal cell death, and found that levels of Bax increase (and Nr13:Bax ratio decreases) with dispersion-induced cell death. However, Nr13 does not by itself appear to protect normal bursal cells from dispersion-induced apoptosis, although Nr13 interacts with Bax in DT40 cells based on coimmunoprecipitation. We have not obtained direct experimental evidence that $\mathrm{Nr} 13$ is able to attenuate the death effects of Bax, and we have not determined whether Bax has any more direct killing mechanism in bursa independent of Bcl2 family members. Currently we are characterizing the chicken bax gene to address these issues.

\section{PMA induction of Nr13}

Inhibition of bursal apoptosis by phorbol esters has been documented (Asakawa et al. 1993; Compton and Waldrip 1998). Phorbol esters activate the protein kinase C (PKC) family, which currently has at least 12 member isoenzymes. The classic PKC- $\alpha$, PKC- $\beta$ I, PKC- $\beta$ II, and PKC- $\gamma$ isoforms are activated by phorbol esters and are calcium dependent. The novel PKC- $\delta$, PKC- $\epsilon$, PKC $\eta$, and PKC- $\theta$ isoforms are calcium independent but activated by phorbol esters. All these isoforms have been linked to apoptosis in different cell lines, but results are conflicting (Deacon et al. 1997). In some systems, PMA treatment induces apoptosis, but in other systems such as the bursa, PMA inhibits apoptosis. We demonstrated by Northern blot analysis that PMA induced Nr13 at the transcriptional level. This induction could contribute to the mechanisms by which PMA acts to block cell death. However, simple overexpression of Nr13 does not by itself block dispersion-induced bursal cell death, indicating that induction of Nr13 is not sufficient to fully explain this effect of PMA. 
Inhibiting bursal apoptosis by $V$-rel or other members of the NF-кB family

$\mathrm{v}-\mathrm{rel}$ is one of the members of the NF-кB complex and is able to transform avian B cells (Neiman et al. 1991; Gilmore et al. 1996). v-rel contains multiple internal mutations and a 118 amino-acid carboxy-terminal deletion compared to c-rel (Sarkar and Gilmore 1993). This group of transcription activators plays an important role in signal transduction and proliferation, and also can either block or enhance apoptosis. Although multiple targets of the NF- $\mathrm{BB}$ factors have been identified /Gilmore et al. 1996), none of them are clearly linked to apoptosis except a recently identified chicken inhibitor-of-apoptosis (IAP) gene (You et al. 1997). We observed here that Nr13 is induced by v-rel, and that both Nr13 gene transcription and v-rel activation of Nr13 expression is most efficient near physiological temperatures for chicken. Sequence analysis of the Nr13 promoter suggests a possible NF-кB binding site (G. Gillet, unpubl.) which could explain why Nr13 is induced by v-rel. These results suggest that v-rel and other factors could inhibit apoptosis through activation of Nr13. However, because expression of Nr13 does not, by itself, block apoptosis induced by dispersion in bursa, Nr13 is not the only determinant of v-rel-mediated inhibition of apoptosis. For example, the previously mentioned chicken IAP is induced by vrel and may by itself, or in combination with Nr13, explain the effect of v-rel on bursal cell apoptosis.

\section{The Nr13 BH4 domain}

Although the functions of the conserved $\mathrm{BH} 1, \mathrm{BH} 2$, and $\mathrm{BH} 3$ domains are important to homodimer or heterodimer formation, the $\mathrm{BH} 4$ domain, which binds at least Raf1 (Wang et al. 1996a), CED4, calcineurin (Shibasaki et al. 1997), and BAG1 (Wang et al. 1996b), may function as a regulatory domain. Deletion of the $\mathrm{BH} 4$ domain of $\mathrm{Bcl} 2$ and the apparent $\mathrm{BH} 4$ helix of $\mathrm{Nr} 13$ both result in loss of the death-protecting function. It was reported that caspase cleaves at an aspartic acid residue immediately carboxy-terminal to the $\mathrm{BH} 4$ domain and converts $\mathrm{Bcl} 2$ into a death agonist (Cheng et al. 1997). Because Bcl2 is considered to function upstream of the caspases, this cleavage could serve as a feedback amplification signal for the death cascade. A comparison of the sequence of Nr13 does not reveal an analogous aspartic acid for caspase cleavage. Moreover, $\mathrm{Bcl} 2$ and $\mathrm{Bcl}-\mathrm{xL}$ both have a long flexible loop structure between the $\mathrm{BH} 4$ and $\mathrm{BH} 3$ domains (Muchmore et al. 1996), which also appears to be much shorter in Nr13. A third difference between $\mathrm{Nr} 13$ and $\mathrm{Bcl} 2$ is the presence of a carboxy-terminal tyrosine residue in $\mathrm{Bcl} 2$, which is important in regulation of $G_{0}-G_{1}$ cell cycle progression (Huang et al. 1997). The $\mathrm{BH} 4$ domain of Nr13 does not have a similar tyrosine residue. Currently we are investigating whether Nr13 can affect cell cycle progression. These structural dissimilarities suggest that Nr13 may have regulatory interactions different from those of $\mathrm{Bcl} 2$ and Bcl-xL. Nr13 might interact with different proteins through the $\mathrm{BH} 4$ domain, and thereby play unique roles that are different from those of other Bcl2 family members.

\section{Other implications of the bursal transplantation experiments}

Overexpression of Nr13 in the chicken bursa did not lead to the development of neoplastic lesions, but did lead to the persistence of transplantable bursal stem cells. The morphology of the primary and secondary reconstituted follicles were normal in appearance. Nr13 overexpression in the bursal follicles might be similar to events in the development of human low-grade follicular lymphomas, which overexpress Bcl2 through a $\mathrm{t}(14,18)$ translocation (Bakhshi et al. 1985). We have not completed long-term observation of transplanted birds. It will be interesting to learn whether the Nr13 overexpression in the bursa will eventually lead to the development of lowgrade lymphomas. Finally, this technique for induced persistence of transplantable stem cells may also provide a useful tool for further investigation and characterization of these stem cells.

\section{Materials and methods}

\section{Cloning of chicken Mcl1 and A1}

Previously unidentified chicken homologs of A1 and Mcl1 were found by random single pass sequencing of an oligo (dT)-primed cDNA library prepared from activated ConA-treated splenic lymphocytes. The complete sequence of these clones was obtained by primer walking. The Mcll clone was incomplete and contained sequence encoding the carboxy-terminal 209 amino acids. This region showed $60 \%$ sequence identity with human Mcl1 (Kozopas et al. 1993), including the highly conserved Bcl2 homology $(\mathrm{BH})$ domains. The A1 cDNA contained the entire coding region and encodes a protein of 174 amino acids that is nearly identical in size (175 amino acids) to human A1 (Karsan et al. 1996). The predicted amino acid sequence shows $46 \%$ overall sequence similarity to the human A1 cDNA with two highly conserved $\mathrm{BH}$ domains. The GenBank accession numbers for chicken Mcll and Al are AS120210 and AS120211, respectively.

\section{Cell culture}

Previously described retroviral packaging lines PE501 and PG13 were grown in DME supplemented with $10 \%$ fetal bovine serum (Miller and Rosman 1989). DT40 bursal lymphoma-derived cells have been described (Baba et al. 1985). DT40 cells were grown in DME supplemented with $10 \%$ BCS, $10 \%$ tryptose phosphate (DIFCO) and $2 \%$ chicken serum (Sigma). For low serum condition experiments, DT40 cells were grown without BCS in DME supplemented with $10 \%$ tryptose phosphate and $1 \%$ CS.

\section{Bursal transplant experiments}

The technique of primary and secondary bursal transplantation has been described (Neiman et al. 1985; Thompson et al. 1987). Briefly, fertilized eggs from inbred SC strain White Leghorn chicken were obtained from Hyline Farms, Dallas Center, IA. Cyclophosphamide, $1.25 \mathrm{mg} /$ day, was injected into chorioallan- 
toic veins on days 15,16 , and 17 to ablate the bursa. Donor bursal cells were obtained from normal 15-day-old embryos or transplanted birds 3-4 weeks after hatching. Donor cells were resuspended in Hams media and dispersed by passing through sterile mesh. The dispersed bursal cells were cocultivated with retroviral vector-producing packaging cells overnight before injected into cyclophosphamide-ablated recipient birds. For secondary transplants, the recipient birds were newly hatched chicks injected with $3 \mathrm{mg}$ of cyclophosphamide for 3 days to ablate the bursal lymphocytes and the donor cells were bursal lymphocytes from 4-week-old primary transplant birds. Transplanted birds were sacrificed at 4 weeks for histological and biochemical analysis. Frozen sections of the bursa were used for GFP detection.

\section{Construction of retroviral vectors and packaging cell lines}

The plasmid pBS-NR with Nr13 cDNA (Gillet et al. 1995) was digested with EcoRI and the 1.6-kb Nr13 fragment was inserted into the polycloning site of pLXSN (Miller and Rosman 1989) or pLXCG retroviral vectors to construct pLNRSN and pLNRCG. pLXCG was derived from pLNCG (Rasko 1997) by replacing the BamHI-XbaI (SV40 promoter-neomycin resistance gene) fragment of LXSN with an analogous (CMV promoter-GFP) fragment from pLNCG. pMH105 plasmid containing v-rel (White and Gilmore 1993) was generously provided by Dr. Thomas Gilmore (Boston University). v-rel was excised with $\mathrm{XbaI}$ from pMH105 and inserted into an $\mathrm{HpaI}$ site of pLXSN vector by blund end cloning. To generate amino-terminal helix deletion of Nr13, pBS-NR was digested with SmaI and NarI, and religated with linker oligonucleotides to create a 60-nucleotide deletion. Junctional sequence was confirmed by automated sequence analysis. All retroviral constructs were transfected into PE501 packaging cells (Miller and Rosman 1989) by calcium phosphate precipitation. The viral-containing supernatants were used to infect PG13 packaging cells (Miller et al. 1991) to establish stable packaging line as described. Viral titers from productive clones were assayed on HeLa cells. Virus-producing clones used for these studies produced titers of $10^{5}-10^{6}$ G418-resistant clones per milliter.

\section{Immunoprecipitation and immunoblotting analysis}

DT40 cells were harvested and washed with cold phosphate buffered saline twice and lysed with buffer containing $50 \mathrm{~mm}$ tris (pH 7.5), $100 \mathrm{~mm} \mathrm{NaCl}, 5$ mm EDTA, 1\% Triton X-100, 1\% NP40, 1 mM PMSF protease inhibitor. The lysate was sonicated and centrifuged for $15 \mathrm{~min}$. The supernatant was mixed with preimmune serum for $30 \mathrm{~min}$. Immunoglobulin was removed by mixing with Pansorbin for 30 min. Pansorbin was removed by centrifugation, and the supernatant was incubated with $\mathrm{Nr} 13$ antiserum at 1:50 dilution for $4 \mathrm{hr}$. Pansorbin was added for another $45 \mathrm{~min}$. The immune complex was precipitated and washed three times with RIPA buffer. SDS loading buffer was added to the washed Pansorbin and boiled $5 \mathrm{~min}$ to solubilize the immune complex before SDS-PAGE analysis. After the electrophoresis, the protein was transferred to a nitrocellulose membrane for standard Western blot analysis using a commercially available anti-Bax antiserum at concentration of 1:100 (Santa Cruz Biotechnology, CA).

\section{Northern blot analysis and RNase protection assay}

RNAs from DT40 cells or bursal tissues were isolated with RNAzol reagent (Tel-Test, Inc., Friendswood, TX). Samples were analyzed on $1 \%$ agarose gel, and transferred to Hybond membranes. Probes were labeled with ${ }^{32} \mathrm{P}$ by random priming to a specific activity $\sim 2 \times 10^{7} \mathrm{cpm} / \mu \mathrm{g}$. The RNase protection probe was cloned in antisense orientation into a Bluescript-KS plasmid (Stratagene, La Jolla, CA). RNA was transcribed in vitro by T7 RNA polymerase using $\left[\alpha^{32} \mathrm{P}\right] \mathrm{UTP}$ as label. RNA samples were hybridized with labeled probe overnight, followed by 10 $\mathrm{mg} / \mathrm{ml} \mathrm{RNase}$ digestion for $45 \mathrm{~min}$. The protected fragments were analyzed by $6 \%$ urea-polyacrylamide gel and autoradiography.

\section{Acknowledgments}

We thank Dr. Thomas Gilmore for the v-rel plasmid, Dr. Dusty Miller for the retroviral vectors and packaging lines, Dr. Cheryl Brown for advice, and Drs. Alanna Ruddell and David Hochenbery for helpful suggestion and discussion. We also thank Will Krespan of DuPont Delaware Technology Park for sequencing of the chicken T cell library. This work is supported by grant CA20068 from the National Cancer Institute.

The publication costs of this article were defrayed in part by payment of page charges. This article must therefore be hereby marked 'advertisement' in accordance with 18 USC section 1734 solely to indicate this fact.

\section{References}

Asakawa, J., V.K. Tsiagbe, and G.J. Thorbecke. 1993. Protection against apoptosis in chicken bursa cells by phorbol ester in vitro. Cell. Immunol. 147: 180-187.

Baba, T.W., B.P. Giroir, and E.H. Humphries. 1985. Cell lines derived from avian lymphomas exhibit two distinct phenotypes. Virology 144: 139-151.

Bakhshi, A., J.P. Jensen, P. Goldman, J.J. Wright, O.W. McBride, A.L. Epstein, and S.J. Korsmeyer. 1985. Cloning the chromosomal breakpoint of $\mathrm{t}(14 ; 18)$ human lymphomas: Clustering around $\mathrm{JH}$ on chromosome 14 and near a transcriptional unit on 18. Cell 41: 899-906.

Cheng, E.H., D.G. Kirsch, R.J. Clem, R. Ravi, M.B. Kastan, A. Bedi, K. Ueno, and J.M. Hardwick. 1997. Conversion of Bcl-2 to a Bax-like death effector by caspases. Science 278: 19661968.

Chinnaiyan, A.M., K. O'Rourke, B.R. Lane, and V.M. Dixit. 1997. Interaction of CED-4 with CED-3 and CED-9: A molecular framework for cell death. Science 275: 1122-1126.

Compton, M.M. and H.M. Waldrip. 1998. Endogenous activation of apoptosis in bursal lymphocytes: Inhibition by phorbol esters and protein synthesis inhibitors. Cell. Immunol. 184: 143-152.

Cryns, V. and J. Yuan. 1998. Proteases to die for. Genes \& Dev. 12: $1551-1570$.

Cryns, V.L., Y. Byun, A. Rana, H. Mellor, K.D. Lustig, L. Ghanem, P.J. Parker, M.W. Kirschner, and J. Yuan. 1997. Specific proteolysis of the kinase protein kinase C-related kinase 2 by caspase-3 during apoptosis. Identification by a novel, small pool expression cloning strategy. J. Biol. Chem. 272: 2944929453.

Deacon, E.M., J. Pongracz, G. Griffiths, and J.M. Lord. 1997. Isoenzymes of protein kinase C: Differential involvement in apoptosis and pathogenesis. Mol. Pathol. 50: 124-131.

Eguchi, Y., D.L. Ewert, and Y. Tsujimoto. 1992. Isolation and characterization of the chicken bcl-2 gene: Expression in a variety of tissues including lymphoid and neuronal organs in adult and embryo. Nucleic Acids Res. 20: 4187-4192.

Evan, G.I., A.H. Wyllie, C.S. Gilbert, T.D. Littlewood, H. Land, 
M. Brooks, C.M. Waters, L.Z. Penn, and D.C. Hancock. 1992. Induction of apoptosis in fibroblasts by c-myc protein. Cell 69: 119-128.

Gillet, G., M. Guerin, A. Trembleau, and G. Brun. 1995. A Bcl2-related gene is activated in avian cells transformed by the Rous sarcoma virus. EMBO I. 14: 1372-1381.

Gilmore, T.D., M. Koedood, K.A. Piffat, and D.W. White. 1996. Rel/NF-kappaB/IkappaB proteins and cancer. Oncogene 13: $1367-1378$.

Hanada, M., C. Aime-Sempe, T. Sato, and J.C. Reed. 1995. Structure-function analysis of Bcl-2 protein. Identification of conserved domains important for homodimerization with Bcl-2 and heterodimerization with Bax. J. Biol. Chem. 270: $11962-$ 11969.

Hayward, W.S., B.G. Neel, and S.M. Astrin. 1981. Activation of a cellular onc gene by promoter insertion in ALV-induced lymphoid leukosis. Nature 290: 475-480.

Horvitz, H.R., S. Shaham, and M.O. Hengartner. 1994. The genetics of programmed cell death in the nematode Caenorhabditis elegans. Cold Spring Harbor Symp. Quant. Biol. 59: 377-385.

Huang, D.C., L.A. O’Reilly, A. Strasser, and S. Cory. 1997. The anti-apoptosis function of Bcl-2 can be genetically separated from its inhibitory effect on cell cycle entry. $E M B O J$. 16: 4628-4638.

Jacobsen, K.A., E. Paramithiotis, D.L. Ewert, and M.J. Ratcliffe. 1996. Apoptotic cell death in the chicken bursa of Fabricius. Adv. Exp. Med. Biol. 406: 155-165.

Jurgensmeier, J.M., Z. Xie, Q. Deveraux, L. Ellerby, D. Bredesen, and J.C. Reed. 1998. Bax directly induces release of cytochrome c from isolated mitochondria. Proc. Natl. Acad. Sci. 95: 4997-5002.

Karsan, A., E. Yee, K. Kaushansky, and J.M. Harlan. 1996. Cloning of human Bcl-2 homologue: Inflammatory cytokines induce human A1 in cultured endothelial cells. Blood 87: 3089-3096.

Kneller, D.G, F.E. Cohen, and R. Langridge. 1990. Improvements in protein secondary structure prediction by an enhanced neural network. J. Mol. Biol. 214: 171-182

Knudson, C.M., K.S. Tung, W.G. Tourtellotte, G.A. Brown, and S.J. Korsmeyer. 1995. Bax-deficient mice with lymphoid hyperplasia and male germ cell death. Science 270: 96-99.

Kozopas, K.M., T. Yang, H.L. Buchan, P. Zhou, and R.W. Craig. 1993. MCL1, a gene expressed in programmed myeloid cell differentiation, has sequence similarity to BCL2. Proc. Nat1. Acad. Sci. 90: 3516-3520.

Kroemer, G. 1997. The proto-oncogene Bcl-2 and its role in regulating apoptosis. Nat. Med. 3: 614-620.

Lin, E.Y., A. Orlofsky, H.G. Wang, J.C. Reed, and M.B. Prystowsky. 1996. A1, a Bcl-2 family member, prolongs cell survival and permits myeloid differentiation. Blood 87:983992.

Mangeney, M., J.R. Schmitt, Y. Leverrier, J. Thomas, J. Marvel, G. Brun, and G. Gillet. 1996. The product of the v-src-inducible gene $\mathrm{nr}-13$ is a potent anti-apoptotic factor. Oncogene 13: $1441-1446$.

Miller, A.D. and G.J. Rosman. 1989. Improved retroviral vectors for gene transfer and expression. Biotechniques 7: 980-982, 984-986, 989-990.

Miller, A.D., J.V. Garcia, N. von Suhr, C.M. Lynch, C. Wilson, and M.V. Eiden. 1991. Construction and properties of retrovirus packaging cells based on gibbon ape leukemia virus. J. Virol. 65: 2220-2224

Miyashita, T. and J.C. Reed. 1995. Tumor suppressor p53 is a direct transcriptional activator of the human bax gene. Cell 80: 293-299.
Muchmore, S.W., M. Sattler, H. Liang, R.P. Meadows, J.E. Harlan, H.S. Yoon, D. Nettesheim, B.S. Chang, C.B. Thompson, S.L. Wong, S.L. Ng, and S.W. Fesik. 1996. X-ray and NMR structure of human Bcl-xL, an inhibitor of programmed cell death. Nature 381: 335-341.

Neiman, P., C. Wolf, P.J. Enrietto, and G.M. Cooper. 1985. A retroviral myc gene induces preneoplastic transformation of lymphocytes in a bursal transplantation assay. Proc. Natl. Acad. Sci. 82: 222-226.

Neiman, P.E., S.J. Thomas, and G. Loring. 1991. Induction of apoptosis during normal and neoplastic B-cell development in the bursa of Fabricius. Proc. Nat1. Acad. Sci. 88: 58575861.

Neiman, P.E., C. Blish, C. Heydt, G. Loring, and S.J. Thomas. 1994a. Loss of cell cycle controls in apoptotic lymphoblasts of the bursa of Fabricius. Mol. Biol. Cell 5: 763-772.

Neiman, P.E., J. Summers, S.J. Thomas, S. Xuereb, and G. Loring. 1994b. Genetic instability and apoptotic cell death during neoplastic progression of v-myc-initiated B-cell lymphomas in the bursa of Fabricius. Cold Spring Harbor Symp. Quant. Biol. 59: 509-515.

Nicholson, D.W. and N.A. Thornberry. 1997. Caspases: Killer proteases. Trends Biochem. Sci. 22: 299-306.

Oltvai, Z.N., C.L. Milliman, and S.J. Korsmeyer. 1993. Bcl-2 heterodimerizes in vivo with a conserved homolog, Bax, that accelerates programmed cell death. Cell 74: 609-619.

Paramithiotis, E. and M.J. Ratcliffe. 1993. Bursa-dependent subpopulations of peripheral B lymphocytes in chicken blood. Eur. J. Immunol. 23: 96-102.

Pink, J.R., M.J. Ratcliffe, and O. Vainio. 1985. Immunoglobulinbearing stem cells for clones of B (bursa-derived) lymphocytes. Eur. J. Immunol. 15: 617-620.

Rasko, J.E.J., H.-P. Kiem, J.C. Morris, R.J. Gottschalk, L.J. Peterson, R.G. Andrews, and A.D. Miller. 1997. Efficient transduction of hematopoietic progenitors using green fluorescent protein (GFP)-containing retroviral vectors with various pseudotypes. Blood 90: 118a.

Reed, J.C. 1997. Double identity for proteins of the Bcl-2 family. Nature 387: 773-776.

Reynaud, C.A., V. Anquez, H. Grimal, and J.C. Weill. 1987. A hyperconversion mechanism generates the chicken light chain preimmune repertoire. Cell 48: 379-388.

Rosse, T., R. Olivier, L. Monney, M. Rager, S. Conus, I. Fellay, B. Jansen, and C. Borner. 1998. Bcl-2 prolongs cell survival after Bax-induced release of cytochrome c. Nature 391: 496499.

Rudin, C.M. and C.B. Thompson. 1997. Apoptosis and disease: Regulation and clinical relevance of programmed cell death. Annu. Rev. Med. 48: 267-281.

Sarkar, S. and T.D. Gilmore. 1993. Transformation by the v-Rel oncoprotein requires sequences carboxy-terminal to the Rel homology domain. Oncogene 8: 2245-2252.

Schlesinger, P.H., A. Gross, X.M. Yin, K. Yamamoto, M. Saito, G. Waksman, and S.J. Korsmeyer. 1997. Comparison of the ion channel characteristics of proapoptotic BAX and antiapoptotic BCL-2. Proc. Natl. Acad. Sci. 94: 11357-11362.

Sedlak, T.W., Z.N. Oltvai, E. Yang, K. Wang, L.H. Boise, C.B. Thompson, and S.J. Korsmeyer. 1995. Multiple Bcl-2 family members demonstrate selective dimerizations with Bax. Proc. Natl. Acad. Sci. 92: 7834-7838.

Shibasaki, F., E. Kondo, T. Akagi, and F. McKeon. 1997. Suppression of signalling through transcription factor NF-AT by interactions between calcineurin and Bcl-2. Nature 386: $728-731$.

Thompson, C.B. and P.E. Neiman. 1987. Somatic diversification of the chicken immunoglobulin light chain gene is limited 
Lee et al.

to the rearranged variable gene segment. Cell 48: 369-378.

Thompson, C.B., E.H. Humphries, L.M. Carlson, C.L. Chen, and P.E. Neiman. 1987. The effect of alterations in myc gene expression on B cell development in the bursa of Fabricius. Cell 51: 371-381.

Toivanen, P. and A. Toivanen. 1973. Bursal and postbursal stem cells in chicken. Functional characteristics. Eur. J. Immunol. 3: 585-595.

Wang, H.G., U.R. Rapp, and J.C. Reed. 1996a. Bcl-2 targets the protein kinase Raf-1 to mitochondria. Cell 87: 629-638.

Wang, H.G., S. Takayama, U.R. Rapp, and J.C. Reed. 1996b. Bcl-2 interacting protein, BAG-1, binds to and activates the kinase Raf-1. Proc. Nat1. Acad. Sci. 93: 7063-7068.

Weill, J.C. and C.A. Reynaud. 1987. The chicken B cell compartment. Science 238: 1094-1098.

White, D.W. and T.D. Gilmore. 1993. Temperature-sensitive transforming mutants of the v-rel oncogene. J. Virol. 67: 6876-6881.

White, D.W., A. Roy, and T.D. Gilmore. 1995. The v-Rel oncoprotein blocks apoptosis and proteolysis of I kappa B-alpha in transformed chicken spleen cells. Oncogene 10: 857-868.

Wyllie, A.H., J.F. Kerr, and A.R. Currie. 1980. Cell death: The significance of apoptosis. Int. Rev. Cytol. 68: 251-306.

Yang, E. and S.J. Korsmeyer. 1996. Molecular thanatopsis: A discourse on the BCL2 family and cell death. Blood 88: 386401.

Yang, E., J. Zha, J. Jockel, L.H. Boise, C.B. Thompson, and S.J. Korsmeyer. 1995. Bad, a heterodimeric partner for Bcl-xL and Bcl-2, displaces Bax and promotes cell death. Cell 80: 285291.

You, M., P.T. Ku, R. Hrdlickova, and H.R. Bose Jr. 1997. chIAP1, a member of the inhibitor-of-apoptosis protein family, is a mediator of the antiapoptotic activity of the v-Rel oncoprotein. Mol. Cell. Biol. 17: 7328-7341.

Zhou, P., L. Qian, K.M. Kozopas, and R.W. Craig. 1997. Mcl-1, a Bcl-2 family member, delays the death of hematopoietic cells under a variety of apoptosis-inducing conditions. Blood 89: 630-643. 


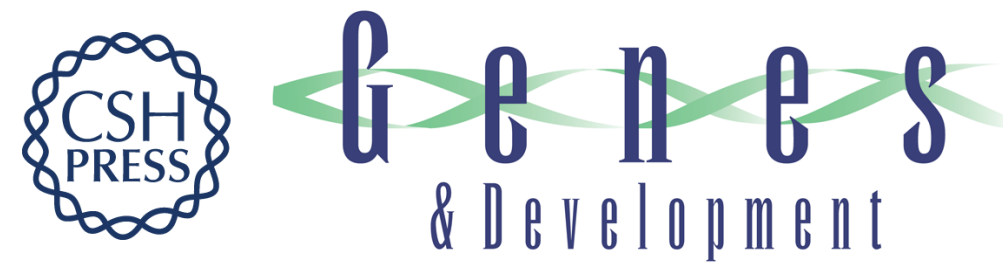

\section{Role of Nr13 in regulation of programmed cell death in the bursa of Fabricius}

Ruey-min Lee, Germain Gillet, Joan Burnside, et al.

Genes Dev. 1999, 13:

References This article cites 60 articles, 26 of which can be accessed free at:

http://genesdev.cshlp.org/content/13/6/718.full.html\#ref-list-1

License

Email Alerting Receive free email alerts when new articles cite this article - sign up in the box at the top Service right corner of the article or click here.

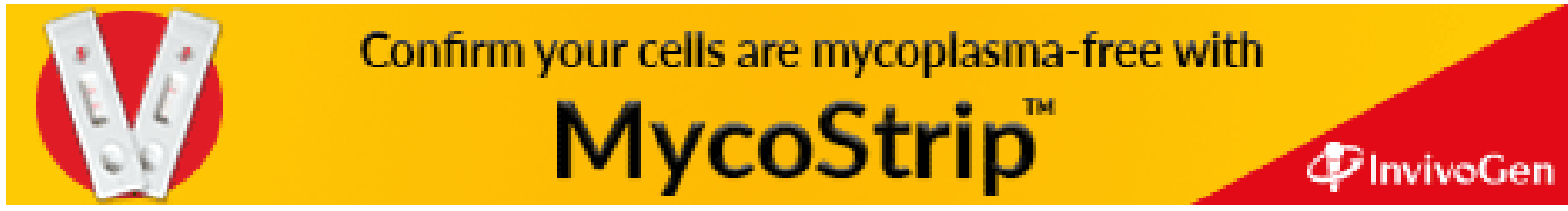

